\title{
ON THE COMPLEXITY OF MIN-MAX OPTIMIZATION PROBLEMS AND THEIR APPROXIMATION*
}

\author{
KER-I KO and CHIH-LONG LIN \\ Department of Computer Science, State University of New York at Stony Brook, \\ Stony Brook, NY 11794.
}

\begin{abstract}
The computational complexity of optimization problems of the min-max form is naturally characterized by $\Pi_{2}^{P}$, the second level of the polynomial-time hierarchy. We present a number of optimization problems of this form and show that they are complete for the class $\Pi_{2}^{P}$. We also show that the constant-factor approximation versions of some of these optimization problems are also complete for $\Pi_{2}^{P}$.
\end{abstract}

\section{Introduction}

Consider an optimization problem of the following form:

Max-A: for a given input $x$, find $\max _{y}\{|y|:(x, y) \in A\}$, where $A$ is a polynomial-time computable set such that $(x, y) \in A$ only if $|y| \leq$ $p(|x|)$ for some polynomial $p$ (we say $A$ is polynomially related). For instance, if $A=\{(G, Q): G=(V, E)$ is a graph and $Q \subseteq V$ is a clique in $G\}$, then Max-A is the well-known maximum clique problem. ${ }^{1}$ It is immediate that the decision version of Max-A, i.e., the problem of determining whether $\max _{y}\{|y|:(x, y) \in A\}$ is greater than or equal to a given constant $K$, is in $N P$. In the past twenty years, a great number of optimization problems of this type have been shown to be $N P$-complete $[3] .^{2}$

Assume that $A \in P, A$ is polynomially related, and that we are given $m$ input instances $x_{1}, \ldots, x_{m}$ and are asked to find

$$
\min _{1 \leq i \leq m} \max _{y}\left\{|y|:\left(x_{i}, y\right) \in A\right\} .
$$

Then, (the decision version of) this problem is still in $N P$, if the instances $x_{1}, \ldots, x_{m}$ are given as input explicitly. However, if $m$ is exponentially large relative to $|x|$ and the instances $x_{1}, \ldots, x_{m}$ have a succinct representation then the complexity of the problem may be higher than $N P$. For instance, consider the following problem MinmaX-Clique: The input to the problem Minmax-Clique is a graph $G=(V, E)$ with its vertices $V$ partitioned into subsets $V_{i, j}, 1 \leq i \leq I, 1 \leq j \leq J$. For any function $t:\{1, \ldots, I\} \rightarrow\{1, \ldots, J\}$, we let $G_{t}$ denote the induced subgraph of $G$ on the vertex set $V_{t}=\bigcup_{i=1}^{I} V_{i, t(i)}$.

\footnotetext{
* Research supported in part by NSF grant CCR 9121472.

1 A subsete $Q \subseteq V$ is a clique of a graph $G=(V, E)$ if $\{u, v\} \in E$ for all $u, v \in Q$.

2 Strictly speaking, their decision versions are shown to be $N P$-complete. In the rest of the paper, we will however use the term $N P$-complete for both the decision and the optimization versions of the problems.
} 
Minmax-Clique: given a graph $G$ with the substructures described above, find $f_{\text {CLIQUE }}(G)=\min _{t} \max _{Q}\left\{|Q|: Q \subseteq V\right.$ is a clique in $\left.G_{t}\right\}$.

Intuitively, the input $G$ represents a network with $I$ components, with each component $V_{i}$ having $J$ subcomponents $V_{i, 1}, \ldots, V_{i, J}$. At any time $t$, only one subcomponent $V_{i, t(i)}$ of each $V_{i}$ is active, and we are interested in the maximum clique size of $G$ for all possible active subgraphs $G_{t}$ of $G$. For people who are familiar with the $N P$-completeness theory, it is easy to see that the problem Minmax-Clique is in $\Pi_{2}^{P}$, the second level of the polynomial-time hierarchy; i.e., the decision problem of determining whether $f_{\mathrm{CLIQUE}}(G) \leq K$, for a given constant $K$, is solvable by a polynomial-time nondeterministic machine with the help of an $N P$-complete set as the oracle. Therefore, it is probably not in NP. Indeed, we will show in Theorem 10 that this problem is complete for $\Pi_{2}^{P}$.

In general, if an input instance $x$ contains an exponential number of subinstances $\left(x_{1}, \ldots, x_{m}\right)$ (called a parameterized input), then the problem of the form

$$
\begin{aligned}
& \text { Minmax-A: for a given parametrized input } x \text {, find } f_{\text {MinmaX-A }}(x)= \\
& \min _{1 \leq t \leq m} \max _{y}\left\{|y|:\left(x_{t}, y\right) \in A\right\},
\end{aligned}
$$

is a natural generalization of the problem MAx-A and its complexity is in $\Pi_{2}^{P}$. In this paper, we present a number of optimization problems of this type and show that they are complete for $\Pi_{2}^{P}$, and hence are not solvable in deterministic polynomial time even with the help of an NP-complete set as the oracle, assuming that the polynomial-time hierarchy does not collapse to $\Delta_{2}^{P}=P^{N P}$. These problems include the generalized versions of the maximum clique problem, the maximum 3dimensional matching problem, the dynamic Hamiltonian circuit problem and the problem of computing the generalized Ramsey numbers.

We remark that although numerous optimization problems have been known to be $N P$-complete, there are relatively fewer natural problems known to be complete for $\Pi_{2}^{P}$ (or, for $\Sigma_{2}^{P}$, the class of complements of sets in $\Pi_{2}^{P}$ ) (cf. $[8,12,13,14]$ ). Our results here demonstrate a number of new $\Pi_{2}^{P}$-complete problems. We hope it could be a basis from which more $\Pi_{2}^{P}$-complete problems can be identified.

In the recent celebrated result of the $P C P$ characterization of $N P$, Arora et al [1] showed that the constant-factor approximation versions of many optimization problems of the form Max-A, including Max-Clique, are also $N P$-complete. It implies that if $P \neq N P$, then there is a constant $\epsilon>0$ such that no polynomialtime algorithm can find for each input $x$ a solution $y$ of size $|y| \geq(1-\epsilon)\left|y^{*}\right|$ such that $(x, y) \in A$, where $y^{*}$ is an optimum solution for $x$. Through a nontrivial generalization, the $P C P$ characterization of $N P$ has been successfully extended to $\Pi_{2}^{P}[2,5,6]$. We apply this characterization to show that some of the problems of the form Minmax-A also have similar nonapproximability property. That is, if $\Pi_{2}^{P} \neq \Delta_{2}^{P}$, then there exists a constant $\epsilon>0$ such that no polynomial-time oracle algorithm using an $N P$-complete set as the oracle can compute, for each $x$, a value $k$ such that $k^{*} /(1+\epsilon) \leq k \leq(1+\epsilon) k^{*}$, where $k^{*}=f_{\text {MINMAX-A }}(x)$. These problems include the min-max versions of the maximum clique problem, the maximum 3dimensional matching problem and the longest circuit problem. 


\section{Definitions}

In this section, we review the notion of $\Pi_{2}^{P}$-completeness, and present the definition of the optimization problems. In the following, we assume that the reader is familiar with the complexity classes $P, N P$ and the notion of $N P$-completeness. For the formal definitions and examples, the reader is referred to any standard text, for instance, [3].

For any string $x$ in $\{0,1\}^{*}$, we denote by $|x|$ the length of $x$. Let $\langle x, y\rangle$ be any pairing function, i.e., a one-to-one mapping from strings $x$ and $y$ to a single string in polynomial time. A well-known characterization of the class $N P$ is as follows: $A \in N P$ if and only if there exists a set $B \in P$ such that for all $x \in\{0,1\}^{*}$,

$$
x \in A \Longleftrightarrow(\exists y,|y| \leq p(|x|))\langle x, y\rangle \in B,
$$

where $p(n)$ is some polynomial depending only on $A$. The complexity class $\Pi_{2}^{P}$ is a natural extension of the class $N P: A \in \Pi_{2}^{P}$ if and only if there exists a set $B \in P$ such that

$$
x \in A \Longleftrightarrow(\forall y,|y| \leq p(|x|))(\exists z,|z| \leq p(|x|))\langle x,\langle y, z\rangle\rangle \in B .
$$

It is obvious that $N P \subseteq \Pi_{2}^{P}$. Between the complexity classes $N P$ and $\Pi_{2}^{P}$ lies the complexity class $\Delta_{2}^{P}$ (or, $P^{N P}$ ) that consists of all problems that are solvable in polynomial time with the help of an $N P$-complete problem as the oracle. Whether $N P=\Delta_{2}^{P}$ and/or $\Delta_{2}^{P}=\Pi_{2}^{P}$ are major open questions in complexity theory.

A decision problem $A$ is $\Pi_{2}^{P}$-complete, if $A \in \Pi_{2}^{P}$, and for every $A^{\prime} \in \Pi_{2}^{P}$, there is a polynomial-time computable function $f$ such that for each $x \in\{0,1\}^{*}, x \in A^{\prime} \Longleftrightarrow$ $f(x) \in A$ ( $f$ is called a reduction from $A^{\prime}$ to $A$ ). There are a few natural problems known to be complete for $\Pi_{2}^{P}$. A standard $\Pi_{2}^{P}$-complete problem that will be used in our proofs is the following generalization of the famous $N P$-complete problem SAT. Suppose that $F$ is a 3 -CNF boolean formula. We write $F(X, Y)$ to emphasize that its variables are partitioned into two sets $X$ and $Y$. For a 3-CNF boolean formula $F(X, Y)$, and for any truth assignments $\tau_{1}: X \rightarrow\{0,1\}$ and $\tau_{2}: Y \rightarrow\{0,1\}$, we write $F\left(\tau_{1}, \tau_{2}\right)$ to denote the formula $F$ with its variables taking the truth values defined by $\tau_{1}$ and $\tau_{2}$. We also write $t c\left(F\left(\tau_{1}, \tau_{2}\right)\right)$ to denote the number of clauses of $F$ that are true to the truth assignments $\tau_{1}$ and $\tau_{2}$.

$\mathrm{SAT}_{2}$ : for a given 3-CNF boolean formula $F(X, Y)$, determine whether it is true that for all truth assignments $\tau_{1}: X \rightarrow\{0,1\}$, there is a truth assignment $\tau_{2}: Y \rightarrow\{0,1\}$ such that $F\left(\tau_{1}, \tau_{2}\right)=1 .^{3}$

Proposition $1 \mathrm{SAT}_{2}$ is $\Pi_{2}^{P}$-complete.

The problem $\mathrm{SAT}_{2}$ may be viewed as (a subproblem of) the decision version of the following optimization problem:

MinmaX-Sat: for a given 3-CNF boolean formula $F(X, Y)$, find $f_{\mathrm{SAT}}(F)=$ $\min _{\tau_{1}: X \rightarrow\{0,1\}} \max _{\tau_{2}: Y \rightarrow\{0,1\}} \operatorname{tc}\left(F\left(\tau_{1}, \tau_{2}\right)\right)$.

\footnotetext{
3 Throughout the paper, we identify 1 with true and 0 with false.
} 
In the following, we introduce some new optimization problems of the min-max form. For each optimization problem, we also list its corresponding decision version. First, we consider the problem Minmax-Sat in the restricted form.

MinmaX-Sat-B: for a given 3-CNF boolean formula $F(X, Y)$ in which each variable occurs in at most $b$ clauses where $b$ is a constant independent of the size of $F$, find $f_{\mathrm{SAT}}(F)$. (Decision version: for an additional input $K>0$, is $\left.f_{\mathrm{SAT}}(F) \geq K ?\right)$

In addition to the problem Minmax-Clique defined in Section 1, we introduce a few more min-max optimization problems that are the generalizations of some famous $N P$-complete optimization problems based on the idea of parameterized inputs. Recall that for a graph $G=(V, E)$ with its vertex set $V$ partitioned into subsets $V_{i, j}, 1 \leq i \leq I, 1 \leq j \leq J$, and for a function $t:\{1, \ldots, I\} \rightarrow\{1, \ldots, J\}$, we let $V_{t}=\bigcup_{i=1}^{I} V_{i, t(i)}$ and let $G_{t}$ be the induced subgraph of $G$ on the vertex set $V_{t}$. The following generalized vertex cover problem is a dual problem of MinmaX-Clique. For a graph $G=(V, E)$, we say that a subset $V^{\prime} \subseteq V$ is a vertex cover if $V^{\prime} \cap\{u, v\} \neq \emptyset$ for all edges $\{u, v\} \in E$.

Maxmin-VC: given a graph $G$ with its vertex set $V$ partitioned into subsets $\left\{V_{i, j}\right\}_{1 \leq i \leq I, 1 \leq j \leq J}$, find $f_{\mathrm{VC}}(G)=\max _{t} \min _{V^{\prime}}\left\{\left|V^{\prime}\right|: V^{\prime} \subseteq V_{t}\right.$ is a vertex cover of $\left.G_{t}\right\}$. (Decision version: Is $f_{\mathrm{VC}}(G) \leq K$ ?)

The following problem is the generalization of the Hamiltonian circuit problem. For a graph $G=(V, E)$ and a subset $V^{\prime} \subseteq V$, we say $G$ has a circuit on $V^{\prime}$ if there is a cycle of $G$ going through each vertex of $V^{\prime}$ exactly once. We say $G$ is Hamiltonian if $G$ has a circuit on $V$.

Minmax-Circuit: given a graph $G$ with its vertex set $V$ partitioned into subsets

$\left\{V_{i, j}\right\}_{1 \leq i \leq I, 1 \leq j \leq J}$, find $f_{\text {CIRCUIT }}(G)=\min _{t} \max _{V^{\prime}}\left\{\left|V^{\prime}\right|: V^{\prime} \subseteq V_{t}, G_{t}\right.$ has a circiut on $\left.V^{\prime}\right\}$. (Decision version: Is $f_{\text {CIRCUIT }}(G) \geq K$ ?)

The next problem is the generalization of the maximum 3-dimensional matching problem. Let $W$ be a finite set and $S$ be a collection of 3-element subsets of $W$. Let $W^{\prime}$ be a subset of $W$. A subset $S^{\prime} \subseteq S$ is called a (3-dimensional) matching in $W^{\prime}$ if all sets $s \in S^{\prime}$ are mutually disjoint, and are contained in $W^{\prime}$. In the following, if $W=\bigcup_{i=1}^{I} \bigcup_{j=1}^{J} W_{i, j}$, and $t$ is a function from $\{1, \ldots, I\}$ to $\{1, \ldots, J\}$, we write $W(t)$ to denote the set $\bigcup_{i=1}^{I} W_{i, t(i)}$.

MinmaX-3DM: given mutually disjoint finite sets $W_{i, j}, 1 \leq i \leq I, 1 \leq j \leq J$, and a set $S$ of 3 -element subsets of $W=\bigcup_{i=1}^{I} \bigcup_{j=1}^{J} W_{i, j}$, find $f_{3 \mathrm{DM}}(W, S)=$ $\min _{t} \max _{S^{\prime}}\left\{\left|S^{\prime}\right|: S^{\prime} \subseteq S, S^{\prime}\right.$ is a matching in $\left.W(t)\right\}$, where $t$ ranges over all functions from $\{1, \ldots, I\}$ to $\{1, \ldots, J\}$. (Decision version: Is $f_{3 \mathrm{DM}}(W, S) \geq K$ ?)

In addition to the above problems based on the idea of parameterized inputs, we consider several natural problems in $\Pi_{2}^{P}$. First, we consider the problem of computing the Ramsey number. For any graph $G=(V, E)$, a function $c: E \rightarrow\{0,1\}$ is called a coloring of $G$ (with two colors). For any complete graph $G$ with a twocolor coloring $c$, a set $Q \subseteq V$ is a monochromatic clique if all edges between vertices in $Q$ are of the same color. Ramsey theorem states that for any positive integer $K$, there exists an integer $n=R_{K}$ such that for all two-colored complete graph $G$ of 
size $n$, there is a monochromatic clique $Q$ of size $K$. To study the complexity of computing the Ramsey function mapping $K$ to the minimum $R_{K}$, we consider the following generalized version. In the following, we say a function $c: E \rightarrow\{0,1, *\}$ is a partial coloring of a graph $G=(V, E)(c(e)=*$ means the edge $e$ is not colored yet). A coloring $c^{\prime}: E \rightarrow\{0,1\}$ is a restriction of a partial coloring $c$, denoted by $c^{\prime} \preceq c$, if $c^{\prime}(e)=c(e)$ whenever $c(e) \neq *$.

Generalized Ramsey Number(GRN): given a complete graph $G=(V, E)$ with a partial coloring $c$, find $f_{\mathrm{GRN}}(G, c)=\min _{c^{\prime} \preceq c} \max _{Q}\{|Q|: Q$ is a monochromatic clique under $\left.c^{\prime}\right\}$. (Decision version: Is $f_{\mathrm{GRN}}(G, c) \geq K$ ?)

Notice that the Ramsey number $R_{K}$ can be found by a binary search for the graph $G$ of the minimum size that has $f_{\mathrm{GRN}}\left(G, c_{0}\right) \geq K$ with respect to the empty coloring $c_{0}$ (i.e., $c_{0}(e)=*$ for all edges $e$ ).

The next two problems are the variations of the Hamiltonian circuit problem. The first problem is to find, from a given digraph and a subset of alterable edges, the length of the longest circuit in any alteration of those edges. Let $G=(V, E)$ be a digraph and $D$ a subset of $E$. We let $G_{D}$ denote the subgraph of $G$ with vertex set $V$ and edge set $(E-D) \cup \operatorname{inv}(D)$, where $\operatorname{inv}(D)=\{(s, t):(t, s) \in D\}$.

Longest Directed Circuit (LDC): given a digraph $G=(V, E)$ and a subset $E^{\prime}$ of $E$, find $f_{\mathrm{LDC}}\left(G, E^{\prime}\right)=\min _{D \subseteq E^{\prime}} \max _{V^{\prime}}\left\{\left|V^{\prime}\right|: V^{\prime} \subseteq V, G_{D}\right.$ has a circuit on $\left.V^{\prime}\right\}$. (Decision version: Is $f_{\mathrm{LDC}}\left(G, E^{\prime}\right) \geq K$ ?)

The next problem is similar to the above problem, but is about the longest circuits in undirected graphs. For simplicity, we formulate it as a special case of its decision version.

Dynamic Hamiltonian Circuit (DHC): given a graph $G=(V, E)$, and a subset $B$ of $E$, determine whether $G_{D}=(V, E-D)$ is Hamiltonian for all subsets $D$ of $B$ with $|D| \leq|B| / 2$.

\section{3. $\Pi_{2}^{P}$-Completeness Results}

All the problems defined in Section 2 can be easily seen belonging to $\Pi_{2}^{P}$. In this section, we show that Minmax-Circuit, GRN and DHC are actually $\Pi_{2}^{P}$-complete. The problems Minmax-Clique and Minmax-3DM will be shown to be $\Pi_{2}^{P}$-complete in Section 5 together with the stronger results that their contant-factor approximation versions are also $\Pi_{2}^{P}$-complete. The $\Pi_{2}^{P}$-completeness of MAXmin-VC is a corollary of that of Minmax-Clique. The proofs for the $\Pi_{2}^{P}$-completeness of the problems Minmax-SAT-B and LDC are much more involved; we prove them in a separate paper [7].

Theorem 2 Minmax-Circuit is complete for $\Pi_{2}^{P}$.

Proof. We construct a reduction from $\mathrm{SAT}_{2}$ to (the decision version of) MinmaxCircuit. The construction is a modification of the reduction from SAT to the Hamiltonian circuit problem. Let $F$ be a 3-CNF boolean formula over variables $X=\left\{x_{1}, \ldots, x_{r}\right\}$ and $Y=\left\{y_{1}, \ldots, y_{s}\right\}$. Assume that $F=C_{1} \wedge C_{2} \wedge \cdots \wedge C_{n}$, where 
each $C_{j}$ is the OR of three literals. We will define a graph $G$ over $18 n+4 r+2 s$ vertices.

For each clause $C_{j}$, we define a subgraph $H_{j}$ of 18 vertices as shown in Figure 1. This subgraph $H_{j}$ will be connected to other parts of the graph $G$ only through the vertices labeled $\alpha_{k}[j]$ and $\beta_{k}[j], k=1,2,3$. Thus, it has the following property: if a Hamiltonian circuit of $G$ enters $H_{j}$ through $\alpha_{k}[j]$ for some $k=1,2,3$, then it must exit at $\beta_{k}[j]$, and visit either one or two or all three rows of $H_{j}$.

In addition to subgraphs $H_{j}$, we have some more vertices: For each variable $x_{i}$ in $X$, we define four vertices: $u_{i, 0}, u_{i, 1}, \bar{u}_{i, 0}, \bar{u}_{i, 1}$. For each variable $y_{i}$ in $Y$, we define two vertices: $v_{i, 0}, v_{i, 1}$.

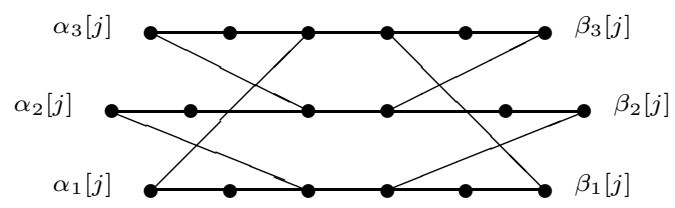

Fig. 1. The subgraph $H_{j}$ for a clause $C_{j}$.

We define the edges between these components as follows.

(1) For each $i, 1 \leq i<r$, we define edges $\left\{u_{i, 1}, u_{i+1,0}\right\},\left\{u_{i, 1}, \bar{u}_{i+1,0}\right\}$, $\left\{\bar{u}_{i, 1}, u_{i+1,0}\right\},\left\{\bar{u}_{i, 1}, \bar{u}_{i+1,0}\right\}$. For $i=r$, we have two edges $\left\{u_{r, 1}, v_{1,0}\right\},\left\{\bar{u}_{r, 1}, v_{1,0}\right\}$.

(2) For each $i, 1 \leq i<s$, we define an edge $\left\{v_{i, 1}, v_{i+1,0}\right\}$. For $i=s$, we have two edges $\left\{v_{s, 1}, u_{1,0}\right\},\left\{v_{s, 1}, \bar{u}_{1,0}\right\}$.

(3) For each literal $z$ and for $k=0,1$, let

$$
w(z)_{k}= \begin{cases}u_{i, k} & \text { if } z=x_{i}, \\ \bar{u}_{i, k} & \text { if } z=\bar{x}_{i}, \\ v_{i, k} & \text { if } z=y_{i} \text { or } \bar{y}_{i} .\end{cases}
$$

Then we define edges to form a path from $w(z)_{0}$ to $w(z)_{1}$ : assume that $z$ occurs as the $k_{1}$ th, $k_{2}$ th $, \ldots, k_{m}$ th literal in clauses $C_{j_{1}}, C_{j_{2}}, \ldots, C_{j_{m}}$, respectively, with $j_{1}<j_{2}<\cdots<j_{m}$. Then we add edges $\left\{w(z)_{0}, \alpha_{k_{1}}\left[j_{1}\right]\right\},\left\{\beta_{k_{m}}\left[j_{m}\right], w(z)_{1}\right\}$, and for each $\ell=1, \ldots, m-1,\left\{\beta_{k_{\ell}}\left[j_{\ell}\right], \alpha_{k_{\ell+1}}\left[j_{\ell+1}\right]\right\}$. (Note that for each pair $\left(u_{i, 0}, u_{i, 1}\right)$ or $\left(\bar{u}_{i, 0}, \bar{u}_{i, 1}\right)$, there is a path between them, and for each pair $\left(v_{i, 0}, v_{i, 1}\right)$ there are two paths between them, corresponding to the occurrences of two literals $y_{i}$ and $\bar{y}_{i}$.)

The above completes the definition of all edges. To complete the reduction, we let $I=r+1, J=2$, and for each $1 \leq i \leq r, V_{i, 0}=\left\{\bar{u}_{i, 0}, \bar{u}_{i, 1}\right\}$ and $V_{i, 1}=\left\{u_{i, 0}, u_{i, 1}\right\}$, and all other vertices are in $V_{r+1,0}=V_{r+1,1}$. (For convenience, we define $V_{i, 0}$ and $V_{i, 1}$ instead of $V_{i, 1}$ and $V_{i, 2}$.) Finally, we let $K=18 n+2 r+2 s$, which is equal to the size of $\left|V_{t}\right|$ for all functions $t:\{1, \ldots, I\} \rightarrow\{0,1\}$.

The correctness of this reduction is very easy to see. We only give a short sketch here. First, assume that for each truth assignment $\tau_{1}$ on $X$, there is a truth assignment $\tau_{2}$ on $Y$ satisfying all clauses $C_{j}$ in $F$. Let $t:\{1, \ldots, r+1\} \rightarrow\{0,1\}$ be any function. Then, $t$ defines a truth assignment $\tau_{1}\left(x_{i}\right)=t(i)$, and all vertices in $V_{i, t(i)}$ corresponds to "true" literals under $\tau_{1}$. From this $\tau_{1}$, there is a truth 
assignment $\tau_{2}$ on $Y$ that satisfies all clauses. Now, for each "true" literal $z$ under $\tau_{1}$ and $\tau_{2}$, we have a path from $w(z)_{0}$ to $w(z)_{1}$ in $G_{t}$. Connecting these paths together forms a Hamiltonian circuit for $G_{t}$, since each subgraph $H_{j}$ is visited by at least one such paths.

Conversely, assume that for any $t:\{1, \ldots, I\} \rightarrow\{0,1\}$, there is a Hamiltonian circuit $\Pi_{t}$ in $G_{t}$. Then, by the basic property of subgraphs $H_{j}$, this circuit $\Pi_{t}$ defines, for each pair of nodes $\left(w(z)_{0}, w(z)_{1}\right)$ in $V_{t}$, a path from $w(z)_{0}$ to $w(z)_{1}$. That is, for each $\tau_{1}$ on $X$ such that $\tau_{1}\left(x_{i}\right)=t(i)$, we can define a truth assignment $\tau_{2}$ on $Y$ by $\tau_{2}\left(y_{i}\right)=1$ (or, $\tau_{2}\left(y_{i}\right)=0$ ) if the path of $\Pi_{t}$ from $v_{i, 0}$ to $v_{i, 1}$ visits nodes corresponding to the literal $y_{i}$ (or, respectively, $\bar{y}_{i}$ ). Since each subgraph $H_{j}$ is visited by at least one of such paths, the assignments $\tau_{1}$ and $\tau_{2}$ together must satisfy each clause $C_{j}$.

Theorem 3 GRN is complete for $\Pi_{2}^{P}$.

Proof. We construct a reduction from $\mathrm{SAT}_{2}$ to GRN. Let $F$ be a 3-CNF formula over variables $X=\left\{x_{1}, \ldots, x_{r}\right\}$ and $Y=\left\{y_{1}, \ldots, y_{s}\right\}$. Assume that $F=C_{1} \wedge C_{2} \wedge \cdots \wedge C_{n}$, where each $C_{i}$ is the OR of three literals. We further assume that $r \geq 2$ and $n \geq 3$. Let $K=2 r+n$. The graph $G$ has $N=6 r+4 n-4$ vertices. We divide them into three groups: $V_{X}=\left\{x_{i, j}, \bar{x}_{i, j}: 1 \leq i \leq r, 1 \leq j \leq 2\right\}, V_{C}=\left\{c_{i, j}: 1 \leq i \leq n, 1 \leq j \leq 3\right\}$ and $V_{R}=\left\{r_{i}: 1 \leq i \leq 2 r+n-4\right\}$. The partial coloring $c$ on the edges of $G$ is defined as follows (we use colors blue and red instead of 0 and 1 ):

(1) The edges among $x_{i, 1}, x_{i, 2}, \bar{x}_{i, 1}$ and $\bar{x}_{i, 2}$, for each $i, 1 \leq i \leq r$, are colored by red, except that the edges $e_{i}=\left\{x_{i, 1}, x_{i, 2}\right\}$ and $\bar{e}_{i}=\left\{\bar{x}_{i, 1}, \bar{x}_{i, 2}\right\}$ are not colored (i.e., $\left.c\left(e_{i}\right)=c\left(\bar{e}_{i}\right)=*\right)$.

(2) All other edges between two vertices in $V_{X}$ are colored by blue; i.e., $c\left(\left\{x_{i, j}\right.\right.$, $\left.\left.x_{i^{\prime}, j^{\prime}}\right\}\right)=c\left(\left\{x_{i, j}, \bar{x}_{i^{\prime}, j^{\prime}}\right\}\right)=$ blue if $i \neq i^{\prime}$.

(3) All edges among vertices in $V_{R}$ are colored by red. red.

(4) For each $i, 1 \leq i \leq k$, the three edges among $c_{i, 1}, c_{i, 2}$ and $c_{i, 3}$ are colored by

(5) The edge between two vertices $c_{i, j}$ and $c_{i^{\prime}, j^{\prime}}$, where $i \neq i^{\prime}$, is colored by red if the $j$ th literal of $C_{i}$ and the $j^{\prime}$ th literal of $C_{i^{\prime}}$ are complementary (i.e., one is $x_{q}$ and the other is $\bar{x}_{q}$, or one is $y_{q}$ and the other is $\bar{y}_{q}$ for some $q$ ). Otherwise, it is colored by blue.

(6) The edge between any vertex in $V_{R}$ and any vertex in $V_{X}$ is colored by red, and the edge between any vertex in $V_{R}$ and any vertex in $V_{C}$ is colored by blue.

(7) For each vertex $c_{i, j}$ in $V_{C}$, if the $j$ th literal of $C_{i}$ is $y_{q}$ or $\bar{y}_{q}$ for some $q$, then all edges between $c_{i, j}$ and any vertex in $V_{X}$ are colored by blue. If the $j$ th literal of $C_{i}$ is $x_{q}$ for some $q$, then all edges between $c_{i, j}$ and any vertex in $V_{X}$, except $\bar{x}_{q, 1}$ and $\bar{x}_{q, 2}$, are colored by blue, and $c\left(\left\{c_{i, j}, \bar{x}_{q, 1}\right\}\right)=c\left(\left\{c_{i, j}, \bar{x}_{q, 2}\right\}\right)=$ red. The case where the $j$ th literal of $C_{i}$ is $\bar{x}_{q}$ for some $q$ is symmetric; i.e., all edges between $c_{i, j}$ and any vertex in $V_{X}$, except $x_{q, 1}$ and $x_{q, 2}$, are colored by blue, and $c\left(\left\{c_{i, j}, x_{q, 1}\right\}\right)=c\left(\left\{c_{i, j}, x_{q, 2}\right\}\right)=$ red.

The above completes the construction of the graph $G$ and its partial coloring $c$. Notice that the partial coloring $c$ has $c(e) \neq *$ for all edges $e$ except $e_{i}$ and $\bar{e}_{i}$, for $1 \leq i \leq r$. Now we prove that this construction is correct. First assume that for 
each assignment $\tau_{1}: X \rightarrow\{0,1\}$, there is an assignment $\tau_{2}: Y \rightarrow\{0,1\}$ such that $F\left(\tau_{1}, \tau_{2}\right)=1$. We verify that for any two-coloring restriction $c^{\prime}$ of $c$, there must be a size- $K$ monochromatic clique $Q$.

We note that if $c^{\prime}\left(e_{i}\right)=c^{\prime}\left(\bar{e}_{i}\right)=$ red for some $i \leq r$, then the vertices $x_{i, 1}$, $x_{i, 2}, \bar{x}_{i, 1}, \bar{x}_{i, 2}$, together with vertices in $V_{R}$, form a red clique of size $\left|V_{R}\right|+4=K$. Therefore, we may assume that for each $i, 1 \leq i \leq r$, at least one of $c^{\prime}\left(e_{i}\right)$ and $c^{\prime}\left(\bar{e}_{i}\right)$ is blue. Now we define an assignment $\tau_{1}$ on $X$ by $\tau_{1}\left(x_{i}\right)=1$ if and only if $c^{\prime}\left(e_{i}\right)=$ blue. For this assignment $\tau_{1}$, there is an assignment $\tau_{2}$ on $Y$ such that each clause $C_{i}$ has a true literal. For each $i, 1 \leq i \leq k$, let $j_{i}$ be the least $j, 1 \leq j \leq 3$, such that the $j$ th literal of $C_{i}$ is true to $\tau_{1}$ and $\tau_{2}$. Let $Q_{C}=\left\{c_{i, j_{i}}: 1 \leq i \leq n\right\}$ and $Q_{X}=\left\{x_{i, j}: c^{\prime}\left(e_{i}\right)=\right.$ blue, $\left.1 \leq j \leq 2\right\} \cup\left\{\bar{x}_{i, j}: c^{\prime}\left(e_{i}\right)=\right.$ red, $\left.1 \leq j \leq 2\right\}$. Let $Q=Q_{C} \cup Q_{X}$. It is clear that $Q$ is of size $2 r+n$. Furthermore, $Q$ is a blue clique: (i) every two vertices in $Q_{C}$ are connected by a blue edge because they both have value true under $\tau_{1}$ and $\tau_{2}$ and so are not complementary; (ii) every two vertices in $Q_{X}$ are connected by a blue edge by the definition of $Q_{X}$, and (iii) if a vertex $c_{i, j_{i}}$ in $Q_{C}$ corresponds to a literal $x_{q}$, then $\tau_{1}\left(x_{q}\right)=1$ and so $\bar{x}_{q, 1}, \bar{x}_{q, 2} \notin Q_{X}$ and hence all the edges between $c_{i, j_{i}}$ and each of $x_{i^{\prime}, j^{\prime}}$ or $\bar{x}_{i^{\prime}, j^{\prime}} \in Q_{X}$ are colored blue.

Conversely, assume that there exists an assignment $\tau_{1}$ on $X$ such that for all assignments $\tau_{2}$ on $Y, F\left(\tau_{1}, \tau_{2}\right)=0$. Then, consider the following coloring $c^{\prime}$ on edges $e_{i}$ and $\bar{e}_{i}: c^{\prime}\left(e_{i}\right)=$ blue and $c^{\prime}\left(\bar{e}_{i}\right)=$ red if $\tau_{1}\left(x_{i}\right)=1$, and $c^{\prime}\left(e_{i}\right)=$ red and $c^{\prime}\left(\bar{e}_{i}\right)=$ blue if $\tau_{1}\left(x_{i}\right)=0$. By the definition of $c^{\prime}$, the largest red clique in $V_{X}$ is of size 3 . Also, the largest red clique in $V_{C}$ is of size 3 , since every edge connecting two noncomplementary literals in two different clauses is colored by blue. Thus, the largest red clique containing $V_{R}$ is of size $K-1$, and the largest red clique containing at least one vertex of $V_{C}$ is of size $\leq 6<K$.

Next, assume by way of contradiction that there is a blue clique $Q$ of $G$ of size $K$. From our coloring, it is clear that, for each $i, 1 \leq i \leq r, Q$ contains exactly two vertices in $\left\{x_{i, 1}, x_{i, 2}, \bar{x}_{i, 1}, \bar{x}_{i, 2}\right\}$, and for each $i, 1 \leq i \leq n, Q$ contains exactly one $c_{i, j_{i}}$, for some $1 \leq j_{i} \leq 3$. Define $\tau_{2}: Y \rightarrow\{0,1\}$ by $\tau_{2}\left(y_{q}\right)=1$ if and only if the $j_{i}$ th literal of $C_{i}$ is $y_{q}$ for some $i, 1 \leq i \leq k$. Then, there is a clause $C_{i}$ such that $C_{i}$ is not satisfied by $\tau_{1}$ and $\tau_{2}$. In particular, the $j_{i}$ th literal of $C_{i}$ is false to $\tau_{1}$ and $\tau_{2}$.

Case 1. The $j_{i}$ th literal of $C_{i}$ is $x_{q}$ for some $q$. Then, $\tau_{1}\left(x_{q}\right)=0$, and so $c^{\prime}\left(e_{q}\right)=$ red, and the edges between $c_{i, j_{i}}$ and each of $\bar{x}_{q, 1}$ and $\bar{x}_{q, 2}$ are red. This contradicts the above observation that $Q$ contains two vertices in $\left\{x_{q, 1}, x_{q, 2}, \bar{x}_{q, 1}, \bar{x}_{q, 2}\right\}$

Case 2. The $j_{i}$ th literal of $C_{i}$ is $\bar{x}_{q}$ for some $q$. This is symmetric to Case 1.

Case 3. The $j_{i}$ th literal of $C_{i}$ is $y_{q}$ for some $q$. This is not possible, because by the definition of $\tau_{2}, \tau_{2}\left(y_{q}\right)=1$, but by the property that $C_{i}$ is not satisfied by $\tau_{1}$ and $\tau_{2}, \tau_{2}\left(y_{q}\right)=0$.

Case 4 . The $j_{i}$ th literal of $C_{i}$ is $\bar{y}_{q}$ for some $q$. Then, $\tau_{2}\left(y_{q}\right)=1$, and hence, by the definition of $\tau_{2}$, there must be another $i^{\prime} \leq n, i^{\prime} \neq i$, such that $c_{i^{\prime}, j_{i^{\prime}}}$ is in $Q$ and the $j_{i^{\prime}}$ th literal of $C_{i^{\prime}}$ is $y_{q}$. So, the edge between $c_{i, j_{i}}$ and $c_{i^{\prime}, j_{i^{\prime}}}$ is colored by red. This is again a contradiction.

The above case analysis shows that there is no blue clique in $G$ of size $K$ either. So the theorem is proven.

Theorem 4 DHC is $\Pi_{2}^{\mathrm{P}}$-complete. 
Proof. We reduce $\mathrm{SAT}_{2}$ to DHC. Let $F=C_{1} \wedge C_{2} \wedge \cdots \wedge C_{n}$ be a boolean formula over variables in $X=\left\{x_{1}, \ldots, x_{r}\right\}$ and $Y=\left\{y_{1}, \ldots, y_{s}\right\}$ where $C_{i}$ 's are three-literal clauses. We construct a graph $G$ from $F$. A basic component of the graph $G$ is a NOT device as shown in Figure 2(a). (It was first introduced in [9], and was called an exclusive-OR device in [11]). Schematically, the two horizontal line paths are represented by two broadened line segments, one designated as input and the other output to the device, and the four vertical paths are "condensed" into one arrow, running from input to output (see Figure 2(c)). As shown in [9], there are only two ways for a Hamiltonian circuit to traverse such a device, one of them indicated in Figure 2(a) by thicker line segments. For convenience, if a NOT device is traversed as shown in Figure 2(a), we say that the NOT device has input true.

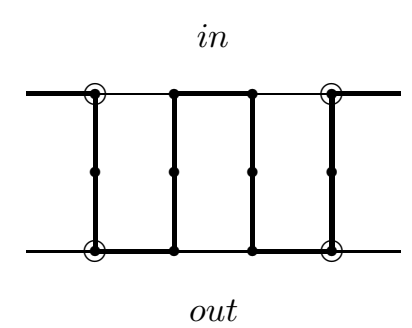

(a)

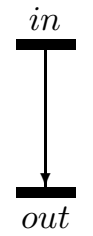

(c)

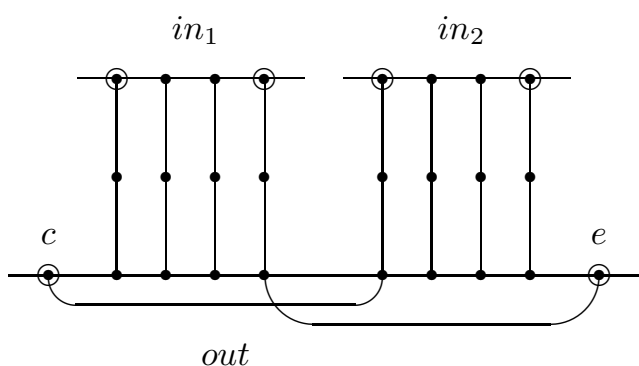

(b)

Fig. 2. The NOT and NAND devices.

Using two NOT devices, we also have the NAND device as shown in Figure 2(b) and 2(d). Here, in order for a Hamiltonian circuit to traverse from $c$ to $e$ or vice versa, at least one of the two input NOT devices have to be false. When using these devices, we require that connections to other parts of the graph $G$ can only go through circled vertices.

The graph $G$ we are going to construct consists of a set of interconnected subgraphs:

(1) For each $x_{i} \in X$, we have a variable subgraph $G_{x}(i)$ as shown in Figure 3(a), and for each $y_{j} \in Y$, a $G_{y}(j)$ as in Figure 3(b). The number of NOT devices 
used in each variable subgraph will be defined in (3) below. In addition, we have a component subgraph $G_{w}$, which is a concatenation of $n+r$ NOT devices as shown in Figure 3(c). Note that each variable or component subgraph has some extra labeled vertices. They are not part of any NOT devices, except that $c$ and $e$ of $G_{x}(i)$ are precisely those in the NAND device shown in figure 2(b).

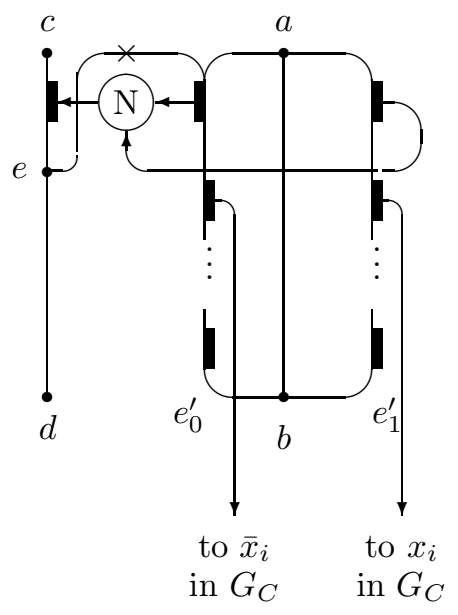

(a)

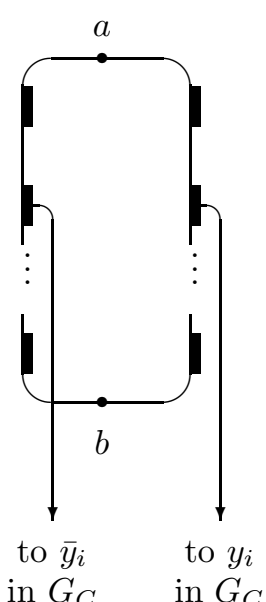

(b)

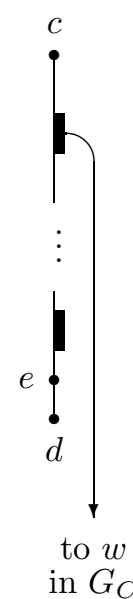

(c)

Fig. 3. The variable subgraphs: (a) $G_{x}(i),(\mathrm{b}) G_{y}(i)$ and (c) $G_{w}$.

(2) For each clause $C_{k}, 1 \leq k \leq n$, we have a clause subgraph $G_{C}(k)$ as shown in Figure 4. In addition, we have $r$ extra clause subgraphs $G_{C}(n+1), \ldots, G_{C}(n+r)$, each of which is a triangle version of Figure 4. Note that if the four conner vertices are further connected as a clique, then any graph containing such a clause subgraph is Hamiltonian only if at least one of the four (or three) NOT devices has its input true.

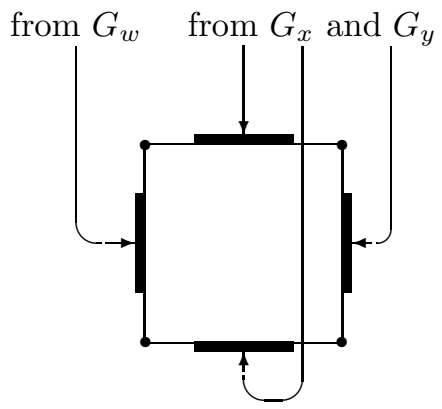

Fig. 4. The clause subgraph $G_{C}(i)$ that has two literals from $X$ and one from $Y$. 
(3) Arrows (of NOT devices) run from variable subgraphs to clause subgraphs as follows:

(a) If literal $\bar{x}_{i}$ (or $x_{i}$ ) occurs in $m$ clauses, then there are $m+2$ NOT devices on the negative (or, respectively, positive) path, the path containing $e_{0}^{\prime}$ (or, respectively, $\left.e_{1}^{\prime}\right)$, of $G_{x}(i)$. The inputs of the first NOT devices on both paths serve as inputs to a NAND device as shown in Figure 3(a).

(b) The numbers of NOT devices in $G_{y}$ 's are defined analogously except that no NAND devices are involved.

(c) An arrow runs from the input of a NOT device in a variable subgraph to the output of a NOT device in a clause subgraph if and only if the corresponding variable, either positive or negative, occurs in that clause. For example, if $C_{k}=\left(x_{i} \vee \cdots\right)$, then there is an arrow running from the input of a NOT device on the positive path of $G_{x}(i)$ to the output of a NOT device in $G_{C}(k)$. Furthermore, for each $G_{C}(n+i), 1 \leq i \leq r$, we have two inputs from $G_{x}(i)$, one corresponding to $x_{i}$ and the other $\bar{x}_{i}$, and another input from one of the NOT devices in $G_{w}$.

(4) Let $a_{x}(i)$ denote the vertex of $G_{x}(i)$ labeled with $a$ in Figure 3(a). Let $b_{x}(i)$, $c_{x}(i), d_{x}(i), e_{x}(i), a_{y}(j), b_{y}(j), e_{0}^{\prime}(i), e_{1}^{\prime}(i), c_{w}, e_{w}$ and $d_{w}$ be defined analogously. Then, in addition to those arrows going to $G_{C}$ from $G_{x}, G_{y}$ and $G_{w}$, these subgraphs are further connected by the following edges (see Figure 5 ):

$\left\langle b_{x}(i), a_{x}(i+1)\right\rangle,\left\langle c_{x}(i), a_{x}(i+1)\right\rangle$ and $\left\langle d_{x}(i), c_{x}(i+1)\right\rangle$ for $1 \leq i \leq r-1$;

$\left\langle b_{y}(j), a_{y}(j+1)\right\rangle$ for $1 \leq j \leq s-1$;

$\left\langle b_{x}(r), a_{y}(1)\right\rangle$ and $\left\langle c_{x}(r), a_{y}(1)\right\rangle$;

$\left\langle d_{x}(r), c_{w}\right\rangle,\left\langle c_{w}, a_{x}(1)\right\rangle$ and $\left\langle d_{w}, c_{x}(1)\right\rangle$;

edges that connect all the corner vertices of all clause subgraphs into a clique;

$\left\langle b_{y}(s), a_{C}\right\rangle,\left\langle b_{C}, e_{w}\right\rangle$, and $\left\langle b_{C}, a_{x}(1)\right\rangle$, where $a_{C}$ and $b_{C}$ are two distinguished corner vertices in two different clause subgraphs.

The graph $G$ is now completed. Finally we define $B$ to be the set of edges $e_{0}^{\prime}(i)$ and $e_{1}^{\prime}(i)$ in $G_{x}(i)$, for all $1 \leq i \leq r$. This finishes the construction.

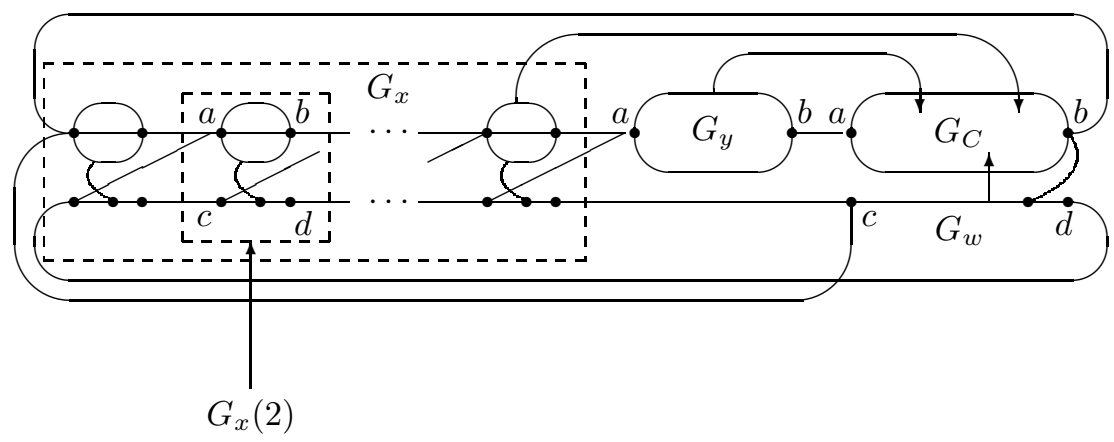

Fig. 5. The interconnection among subgraphs.

We now show that the above construction is a reduction from $\mathrm{SAT}_{2}$ to DHC. First suppose that $F \in \mathrm{SAT}_{2}$. We claim that $G_{D}$ is Hamiltonian for any $D \subseteq B$ with $|D| \leq|B| / 2$. Consider the following two cases: 
Case 1. For some $i, 1 \leq i \leq r$, neither $e_{0}^{\prime}(i)$ nor $e_{1}^{\prime}(i)$ is in $D$. We show the existence of a Hamiltonian circuit $H$ in this case. Let $i$ be the smallest index affirming the case. Starting from $a_{x}(1), H$ visits all vertices in $G_{D}$ in the following order:

(i) $\quad H$ first visits $a_{x}(1), b_{x}(1), a_{x}(2), b_{x}(2), \ldots, a_{x}(i-1), b_{x}(i-1)$ and then $a_{x}(i)$.

(ii) It clockwisely visits all the NOT devices on the "loop" of $G_{x}(i)$, making the inputs to these NOT devices true, take the edge marked with $\times$ (see Figure 3(a)) to $e_{x}(i)$ and leaves at $d_{x}(i)$.

(iii) It then proceeds to visit $c_{x}(i+1), d_{x}(i+1), \ldots, c_{x}(r), d_{x}(r), c_{w}, d_{w}, c_{x}(1)$, $d_{x}(1), \ldots, c_{x}(i-1), d_{x}(i-1), c_{x}(i)$. While going from each $c_{x}(k)$ to $d_{x}(k)$, $k \neq i$, it traverses the NAND device in between (and so sets both inputs false), and from $c_{w}$ to $d_{w}$, sets all the NOT devices in between true.

(iv) It then visits $a_{x}(i+1), b_{x}(i+1), \ldots, a_{x}(r), b_{x}(r)$, and then sets all $y_{j}$ to false (i.e., visits all the NOT devices on the left half of each $G_{y}(j)$ ).

(v) $H$ finally visits $G_{C}$ completely, starting at $a_{C}$ and leaving via $b_{C}$, and returns to $a_{x}(1)$. Note that we are able to do so because all clause subgraphs contain the output of a NOT device whose input is in $G_{w}$, which had been set to true previously; further, all NOT devices in variable subgraphs not traversed before are visited here.

Case 2. Exactly one of $e_{0}^{\prime}(i)$ and $e_{1}^{\prime}(i)$ is in $D$ for all $i, 1 \leq i \leq r$. The Hamiltonian circuit $H$ in this case is as follows. It starts from $a_{x}(1)$.

(i) It chooses the positive (negative) path of $G_{x}(i)$ if $e_{1}^{\prime}(i)$ (respectively, $e_{0}^{\prime}(i)$ ) is not in $D$, and visits all the NOT devices on the path.

(ii) Since the edges $e_{0}^{\prime}(i)$ or $e_{1}^{\prime}(i)$ not in $D$ correspond to a truth assignment $\tau_{1}$ on $X$ (i.e., $\tau_{1}\left(x_{i}\right)=1$ if and only if $e_{1}^{\prime}(i)$ is not in $D$ ), there exists a truth assignment $\tau_{2}$ on $Y$ such that $F\left(\tau_{1}, \tau_{2}\right)$ is true. $H$ sets each $y_{j}$ accordingly by traversing the positive (or negative) path of $G_{y}(j)$ if $y_{j}$ is set to true (or, respectively, false).

(iii) $H$ then traverses $G_{C}$ completely, leaving via $b_{C}$. We are able to do so because $F\left(\tau_{1}, \tau_{2}\right)$ is true and so each $G_{C}(j), 1 \leq j \leq n$, has at least one NOT device having the true input. Further, for each $G_{C}(n+i), 1 \leq i \leq r$, one of the NOT devices corresponding to $x_{i}$ or $\bar{x}_{i}$ has its input true. All NOT devices in variable subgraphs not traversed previously are done at this stage.

(iv) It visits $e_{w}$, then visits $d_{w}, c_{x}(1), d_{x}(1), \ldots, c_{x}(r)$, and finally $d_{x}(r)$, traversing each NAND device between $c_{x}(k)$ and $e_{x}(k)$, since exactly one input has been set true. It leaves via $c_{w}$ and finishes at $a_{x}(1)$.

Since $|D| \leq|B| / 2$, we cannot have $e_{0}^{\prime}(i)$ and $e_{1}^{\prime}(i)$ both in $D$ without having $e_{0}^{\prime}\left(i^{\prime}\right)$ and $e_{1}^{\prime}\left(i^{\prime}\right)$ both not in $D$ for some $i^{\prime}$. Therefore, the above two cases are exhaustive.

Suppose on the other hand that for some truth assignment $\tau_{1}$ on $X, F\left(\tau_{1}, Y\right)$ is not satisfiable. We let $e_{0}^{\prime}(i) \in D$ if $t\left(x_{i}\right)=1$, and $e_{1}^{\prime}(i) \in D$ otherwise. We need to show that $G_{D}$ is not Hamiltonian. Suppose, for the sake of contradiction, that $G_{D}$ has a Hamiltonian circuit $H$. First we can exclude the possibility that $H$ enters and leaves a NOT device (including those within an NAND device) on different sides. Thus we can virtually "ignore" those arrows as far as $H$ is concerned. As a consequence, either all the NOT devices in $G_{w}$ are set to true by $H$ or all set to false by $H$. We consider these two cases separately. 
Case 1. All NOT devices in $G_{w}$ are set to true by $H$. Orientation ignored, we assume that $H$ starts from $c_{w}$, visiting all NOT devices, and then $e_{w} . H$ then has to take $d_{w}$ since taking $b_{C}$ will leave $d_{w}$ unvisited. Next it must visit $c_{x}(1)$, the only choice.

To visit other parts of $G_{D}, H$ eventually has to leave the bottom loop which contains all the distinguished vertices labeled with $c, e$ or $d$. It cannot leave from a vertex $e_{x}(j)$ since this would keep $d_{x}(j)$ out of the reach. Therefore, it must leave at $c_{x}(k)$ for some $1 \leq k \leq r$. It then has to visit $e_{x}(k)$ later. It means that the two inputs to the NAND device between $c_{x}(k)$ and $e_{x}(k)$ must be set to true, which is impossible because only one of $e_{0}^{\prime}(k)$ and $e_{1}^{\prime}(k)$ is in $G_{D}$.

Case 2. All of the NOT devices in $G_{w}$ are set to false by $H$. Orientation ignored, we assume that $H$ starts at $a_{x}(1)$, visiting each variable subgraph. There are two possibilities:

(a) If during traversing $G_{x}(i), H$ enters the bottom loop at either $c_{x}(i)$ or $e_{x}(i)$, then it has to leave the loop before visiting $d_{x}(r)$; otherwise, it would have to visit $c_{w}$ and then either ends its journey prematurally or visits $G_{w}$, contradictary to our assumption. A contradiction can be derived as in Case 1 .

(b) $H$ visits all variable subgraphs in a normal manner (i.e., not going to the bottom loop from $G_{x}(i)$ 's). Then $H$ defines a truth assignment on $X$ and $Y$ which is consistent with $\tau_{1}$ when restricted to $X$. By asumption, at least one of the clause $C_{k}$ of $F$ is not satisfied, meaning that none of the NOT devices of $G_{C}(k)$ has true input. As indicated previously, $H$ then cannot traverse $G_{C}(k)$ completely, which is a contradiction.

\section{Approximation Problems and Their Hardness}

We first formalize the notion of approximating optimization problems. Garey and Johnson [3] have defined the notion of approximating optimization problems of the form Max-A. Since our min-max optimization problems have two parameters, their definition is not suitable to our case. In the following, we define a simple notion of approximating a function. Let $\mathbf{Q}^{+}$be the set of positive rationals and $\mathbf{R}^{+}$the set of positive reals.

Definition 5 Let $f, g:\{0,1\}^{*} \rightarrow \mathbf{Q}^{+}$and $c: \mathbf{N} \rightarrow \mathbf{R}^{+}, c(n)>1$ for all $n$, be given. We say that $g$ approximates $f$ to within a factor of $c$ (c-approximates $f$ in short) if for all $x \in\{0,1\}^{*}$, we have $f(x) / c(|x|)<g(x)<c(|x|) \cdot f(x)$. The $c$-approximation problem of $f$ is to compute a function $g$ that c-approximates $f$.

To define the notion of completeness of $c$-approximation problem of a function $f$, we generalize the notion of reductions between decision problems. In the following, we write $\langle A, B\rangle$ to denote a pair of sets over $\{0,1\}$ with $A \cap B=\emptyset$.

Definition 6 (a) A pair $\langle A, B\rangle$ is polynomial-time separable (or, simply, $\langle A, B\rangle \in$ $P)$ if there exists a set $C \in P$ such that $A \subseteq C$ and $B \subseteq \bar{C}$.

(b) For any two pairs $\langle A, B\rangle$ and $\left\langle A^{\prime}, B^{\prime}\right\rangle$, we say that $\langle A, B\rangle$ is $G$-reducible to $\left\langle A^{\prime}, B^{\prime}\right\rangle$ if there is a polynomial-time computable function $f$ such that $f(A) \subseteq A^{\prime}$ 
and $f(B) \subseteq B^{\prime}$. Let $\mathcal{C}$ be a complexity class. We say that $\langle A, B\rangle$ is $\mathcal{C}$-hard if there exists a set $C$ that is $\mathcal{C}$-hard and $\langle C, \bar{C}\rangle$ is $G$-reducible to $\langle A, B\rangle$.

It is clear that if $P \neq \mathcal{C}$ and that $\langle A, B\rangle$ is $\mathcal{C}$-hard, then $\langle A, B\rangle$ is not polynomialtime separable.

For any functions $s, l:\{0,1\} \rightarrow \mathbf{Q}^{+}$such that $s(x)<l(x)$, we write $\langle f: l(x), s(x)\rangle$ to dentoe the pair of sets $\langle\{x \mid f(x) \geq l(x)\},\{x \mid f(x) \leq s(x)\}\rangle$. The following proposition relates the hardness of approximating functions to that of pairs of decision problems.

Proposition 7 Let $c: \mathbf{N} \rightarrow \mathbf{Q}^{+}, c(n)>1$ for all $n \geq 0$, be polynomial-time computable. Let $s, l:\{0,1\}^{*} \rightarrow \mathbf{Q}^{+}$be two polynomial-time computable functions satisfying $c(|x|) s(x)<l(x) / c(|x|)$. If $\langle f: l(x), s(x)\rangle$ is not polynomial-time separable, then the c-approximation problem of $f$ is not computable in polynomial time.

Proof. Assume that $g$ is a function $c$-approximating $f$. Then, for any $x,|x|=n$, if $f(x) \geq l(x)$, then $g(x)>l(x) / c(n)$; if $f(x) \leq s(x)$, then $g(x)<c(n) s(x)<l(x) / c(n)$. Thus, from $g(x)$ and $l(x) / c(|x|)$, we can tell an instance in $\{x: f(x) \geq l(x)\}$ from an instance in $\{x: f(x) \leq s(x)\}$.

Based on the above proposition, we define the notion of hardness of $C$ approximation problems as follows:

Definition 8 Let $f:\{0,1\}^{*} \rightarrow \mathbf{Q}^{+}$be a given function and $c: \mathbf{N} \rightarrow \mathbf{Q}^{+}, c(n)>1$ be a polynomial-time computable function. We say that the c-approximation problem of $f$ is $\mathcal{C}$-hard if there exist polynomial-time computable functions $s, l:\{0,1\}^{*} \rightarrow \mathbf{Q}^{+}$, $s(x)<l(x)$, such that

1. for all $x$ of length $n, c(n) s(x)<l(x) / c(n)$; and

2. $\langle f: l(x), s(x)\rangle$ is $\mathcal{C}$-hard.

We say the $c$-approximation problem of MinMAX-A is $\Pi_{2}^{P}$-complete if the decision version of MinMAX-A is in $\Pi_{2}^{P}$ and the $c$-approximation problem of $f_{\text {MINMAX-A }}$ is $\Pi_{2}^{P}$-hard.

Remark. In practice, we often prove $\mathcal{C}$-hardness of $\langle f: l(x), s(x)\rangle$, where $l(x)=$ $l \cdot \operatorname{size}(x), s(x)=s \cdot \operatorname{size}(x)$ for some simple function $\operatorname{size}(x)$ and constants $0<s<$ $l \leq 1$. From Proposition 7 , it follows that the $(l / s)^{1 / 2}$-approximation problem for $f$ is $\mathcal{C}$-hard. The function $\operatorname{size}(x)$ is not necessarily the natural size of the instance $x$. Rather, it is a measure designed to prove the hardness of approximation. In the case of Minmax-SAT, a 3 -CNF boolean formula $F$ has $\operatorname{size}(F)=|F|$, the number of clauses in $F$.

The recent breakthrough of Arora et al [1] on the NP-hardness of many optimixation problems in the form of MAX-A is based on a characterization of $N P$ in terms of the notion of probabilistically checkable proofs $(P C P)$. Through a generalization of the notion of $P C P$, this characterization has been extended to the class $\Pi_{2}^{P}$ (it was implicit in [2], and explicit in [6] and [5]). A consequence of this characterization is that the $c$-approximation problem of MinMAX-SAT is $\Pi_{2}^{P}$-complete for some constant $c>0$. This will be our basis for proving other $\Pi_{2}^{P}$-complete $c$-approximation problems. 
Proposition 9 There exists a constant $0<\epsilon<1$ such that $\left\langle f_{\mathrm{SAT}}:|F|,(1-\epsilon)|F|\right\rangle$ is $\Pi_{2}^{P}$-hard. Therefore, there is a constant $c>1$ such that the c-approximation problem for $f_{\mathrm{SAT}}$ is $\Pi_{2}^{P}$-complete.

\section{Nonapproximability Results}

Our proofs of the $\Pi_{2}^{P}$-completeness results for $c$-approximation problems Minmax-A will be done by G-reductions from Minmax-Sat to Minmax-A. More precisely, we will construct G-reductions from $\left\langle f_{\mathrm{SAT}}:|F|,(1-\epsilon)|F|\right\rangle$ to a pair $\left\langle f_{\text {MINMAX-A }}\right.$ : $\left.\left(1-\epsilon_{2}\right) \operatorname{size}(x),\left(1-\epsilon_{1}\right) \operatorname{size}(x)\right\rangle$, where $\epsilon_{1}>\epsilon_{2} \geq 0$. For the proofs below, $\epsilon_{2}$ are always 0. However, in [7], the G-reductions from Minmax-Sat to MinmaX-Sat-B and LDC have $\epsilon_{2}>0$.

We first present the proof that the decision version of the problem Minmax-Clique is $\Pi_{2}^{P}$-complete. The result on the approximation version follows as a corollary.

Theorem 10 Minmax-Clique is $\Pi_{2}^{P}$-complete.

Proof. We reduce the problem $\mathrm{SAT}_{2}$ to Minmax-Clique. Let $F$ be a 3-CNF formula over variables $X=\left\{x_{1}, \ldots, x_{r}\right\}$ and $Y=\left\{y_{1}, \ldots, y_{s}\right\}$. Let $F=C_{1} \wedge \ldots \wedge C_{n}$, where each $C_{i}$ is the OR of three literals. We may assume that each clause $C_{i}$ has at most one literal in $\left\{x_{1}, \ldots, x_{r}, \bar{x}_{1}, \ldots, \bar{x}_{r}\right\}$ (called $X$-literals). Otherwise, we can convert a clause $C_{i}$ of two $X$-literals to two clauses each with one $X$-literal without changing the membership in $\mathrm{SAT}_{2}$. For instance, if $C_{i}=x_{1} \vee x_{2} \vee y_{1}$, then we replace $C_{i}$ with $C_{i, 1}=x_{1} \vee y_{1} \vee z$ and $C_{i, 2}=x_{2} \vee y_{1} \vee \bar{z}$, where $z$ is a new variable not in $X \cup Y$, and it can be checked that the resulting formula $F^{\prime}(X, Y \cup\{z\})$ is in $\mathrm{SAT}_{2}$ if and only if $F(X, Y)$ is in $\mathrm{SAT}_{2}$. (A clause $C_{i}$ with $3 X$-literals is trivially false for some $\tau_{1}$.) We now describe the construction of the graph $G$.

The vertex set $V$ of $G$ is partitioned into $2 n$ subsets $V_{i, j}, 1 \leq i \leq n, j=0,1$ (i.e., $I=n, J=2$ ). For each $1 \leq i \leq n$ and $0 \leq j \leq 1, V_{i, j}$ has 3 vertices $a_{i, j}[k]$, $k=1,2,3$, corresponding to the 3 literals of $C_{i}$, together with $n^{\prime}=\lceil n / 2\rceil$ other vertices $b_{i, j}[k], k=1, \ldots, n^{\prime}$. Let $B_{i, j}=\left\{b_{i, j}[k]: k=1, \ldots, n^{\prime}\right\}$. Within $B_{i, j}$, all vertices are connected (and so $B_{i, j}$ is a clique of size $n^{\prime}$ ). There is no other edge within $V_{i, j}$, and there is no edge between $V_{i, 0}$ and $V_{i, 1}$.

To define the edges between the vertices in $V_{i, j}$ and vertices in $V_{i^{\prime}, j^{\prime}}$ with $i \neq i^{\prime}$, we associate an $X$-literal $x l\left(V_{i, j}\right)$ to each $V_{i, j}$. Each $C_{i}$ has at most one $X$-literal. Suppose $C_{i}$ has an $X$-literal; then we let $x l\left(V_{i, 1}\right)$ be the literal in $C_{i}$ and $x l\left(V_{i, 0}\right)$ be its complement. Suppose $C_{i}$ has no $X$-literals; then we add a dummy variable $x_{r+1}$ and let $x l\left(V_{i, 0}\right)=x l\left(V_{i, 1}\right)=x_{r+1}$. In addition, we define the following terms on vertices $a_{i, j}[k]$ : A vertex $a_{i, j}[k]$ is negative if it corresponds to a $X$-literal in $C_{i}$ and $j=0$. Two vertices $a_{i, j}[k]$ and $a_{i^{\prime}, j^{\prime}}\left[k^{\prime}\right]$ are complementary if they correspond to two complementary literals, i.e., one is $x_{k}$ (or, $y_{k}$ ) and the other is $\bar{x}_{k}$ (or, respectively, $\bar{y}_{k}$ ) for some $k$.

Now, we define edges between $V_{i, j}$ and $V_{i^{\prime}, j^{\prime}}$ with $i \neq i^{\prime}$ as follows:

(1) If $x l\left(V_{i, j}\right)$ and $x l\left(V_{i^{\prime}, j^{\prime}}\right)$ are complementary, then we connect all vertices between $B_{i, j}$ and $B_{i^{\prime}, j^{\prime}}$. There is no other edge between $V_{i, j}$ and $V_{i^{\prime}, j^{\prime}}$. 
(2) If not (1), then there is no edge between $B_{i, j}$ and $V_{i^{\prime}, j^{\prime}}$ and no edge between $B_{i^{\prime}, j^{\prime}}$ and $V_{i, j}$. For any two vertices $a_{i, j}[k]$ and $a_{i^{\prime}, j^{\prime}}\left[k^{\prime}\right]$, they are connected by an edge if and only if they are nonnegative and noncomplementary.

The above completes the graph $G$. Now we prove that this construction is correct with respect to the bound $K=n$. It suffices to prove the following stronger statement:

Claim. If $f_{\mathrm{SAT}}(F)>\lceil|F| / 2\rceil$, then $f_{\mathrm{CLIQUE}}(G)=f_{\mathrm{SAT}}(F)$.

Proof of Claim. First assume that for each truth assignment $\tau_{1}$ on $X$, there is a truth assignment $\tau_{2}$ on $Y$ that satisfies $k^{*}$ clauses of $F$ (i.e., $f_{S A T}(F)=k^{*}$ ). Let $t$ be any mapping from $\{1, \ldots, n\}$ to $\{0,1\}$. First, if for some $i \neq i^{\prime}, x l\left(V_{i, t(i)}\right)$ and $x l\left(V_{i^{\prime}, t\left(i^{\prime}\right)}\right)$ are complementary, then we get a clique $B_{i, t(i)} \cup B_{i^{\prime}, t\left(i^{\prime}\right)}$ that is of size $\geq n \geq k^{*}$. Second, if for all $i \neq i^{\prime}, x l\left(V_{i, t(i)}\right)$ and $x l\left(V_{i^{\prime}, t\left(i^{\prime}\right)}\right)$ are noncomplementary, then there is a unique truth assignment $\tau_{1}$ on $X$ such that $\tau_{1}\left(x l\left(V_{i, t(i)}\right)\right)=1$ for all $i, 1 \leq i \leq n$. (We always let $\tau_{1}$ on the dummy variable $x_{r+1}$ be 1 .) For this assignment $\tau_{1}$, there is a truth assignment $\tau_{2}$ on $Y$ that satisfies $k^{*}$ clauses. Let $I_{T}=\left\{i: C_{i}\left(\tau_{1}, \tau_{2}\right)=1\right\}$. For each $i \in I_{T}$, pick a vertex $a_{i, t(i)}[k]$ that corresponds to a true literal in $C_{i}$ under $\tau_{1}$ and $\tau_{2}$. Note that if we picked a vertex $a_{i, t(i)}[k]$ that corresponds to the $X$-literal in $C_{i}$, then $t(i)$ must be equal to 1 . Thus, it is easy to check that these vertices $a_{i, t(i)}[k]$ form a clique of size $k^{*}$ : (i) if $a_{i, t(i)}[k]$ corresponds to an $X$-literal, then as observed above $t(i)=1$ and it is nonnegative; (ii) no two selected vertices are complementary, since the corresponding literals must be noncomplementary to be satisfied by $\tau_{1}$ and $\tau_{2}$.

Conversely, assume that the maximum clique size of all $G_{t}$ for all $t:\{1, \ldots, n\} \rightarrow$ $\{0,1\}$ is at least $k^{*}$. Let $\tau_{1}$ be any truth assignment on $X$. We need to show that there is a truth assignment $\tau_{2}$ on $Y$ that satisfies $k^{*}$ clauses. Define a mapping $t$ : $\{1, \ldots, n\} \rightarrow\{0,1\}$ by $t(i)=1$ if and only if $\tau_{1}\left(x l\left(V_{i, 1}\right)\right)=1$, i.e., $\tau_{1}\left(x l\left(V_{i, t(i)}\right)\right)=1$ for all $i \leq n$ (we assume that $\tau_{1}\left(x_{r+1}\right)=1$ ). Thus, no two $X$-literals $x l\left(V_{i, t(i)}\right)$ and $x l\left(V_{i^{\prime}, t\left(i^{\prime}\right)}\right)$ are complementary, and so there is no edge between $B_{i, t(i)}$ and $V_{i^{\prime}, t\left(i^{\prime}\right)}$ if $i \neq i^{\prime}$. It follows that the maximum clique $Q$ of $G_{t}$ must consist of a single vertex $a_{i, t(i)}[k]$ in $V_{i, t(i)}$, for $k^{*}$ indices $i$. Let $I_{Q}=\left\{i: Q \cap V_{i, t(i)} \neq \emptyset\right\}$. Now, we define a truth assignment $\tau_{2}$ on $Y$ as follows: if $y_{\ell}$ ever occurs as a literal corresponding to some vertex in the clique $Q$, then assign $\tau_{2}\left(y_{\ell}\right)=1$; otherwise, assign $\tau_{2}\left(y_{\ell}\right)=0$. We check that $\tau_{1}$ and $\tau_{2}$ satisfy $C_{i}$ for all $i \in I_{Q}$. In particular, for each $i \in I_{Q}$, the literal corresponding to the vertex $a_{i, t(i)}[k]$ in $V_{i, t(i)} \cap Q$ must be true to $\tau_{1}$ and $\tau_{2}$ :

(1) If $a_{i, t(i)}[k]$ corresponds to an $X$-literal and it belongs to the clique $Q$, then it must be nonnegative and so $t(i)=1$. That means the corresponding $X$-literal is the same as $x l\left(V_{i, 1}\right)$ and has the value 1 under $\tau_{1}$.

(2) If $a_{i, t(i)}[k]$ corresponds to a $Y$-literal $y_{\ell}$, then since $y_{\ell}$ occurs in $Q, \tau_{2}\left(y_{\ell}\right)=1$.

(3) If $a_{i, t(i)}[k]$ corresponds to a $Y$-literal $\bar{y}_{\ell}$, then $y_{\ell}$ does not occur in the clique $Q$, because $y_{\ell}$ and $\bar{y}_{\ell}$ are complementary and so they cannot be connected. This implies that $\tau_{2}\left(\bar{y}_{\ell}\right)=1$.

The above completes the proof of the claim and hence the correctness of the reduction.

Corollary 11 There exists a constant $c>1$ such that the c-approximation problem of Minmax-Clique is $\Pi_{2}^{P}$-complete. 
Proof. Let $g$ be the reduction of the above theorem. In the above proof, we showed that for any 3 -CNF formula $F, f_{\mathrm{SAT}}(F)=f_{\mathrm{CLIQUE}}(g(F))$ as long as $f_{\mathrm{SAT}}(F)>$ $\lceil|F| / 2\rceil$. For any graph $G$ whose vertex set $V$ is partitioned into $V_{i, j}, 1 \leq i \leq I$, $1 \leq j \leq J$. We let $\operatorname{size}(G)=I$. Then the above observation implies that $g$ is a G-reduction from $\left\langle f_{\mathrm{SAT}}:|F|,(1-\epsilon)|F|\right\rangle$ to $\left\langle f_{\mathrm{CLIQUE}}: \operatorname{size}(G),(1-\epsilon) \operatorname{size}(G)\right\rangle$.

We note that the $\Pi_{2}^{P}$-completeness of MAxMin-VC follows from that of Minmax-Clique since they are the dual problems to each other. However, the $c$-approximation results do not carry over.

Corollary 12 Maxmin-VC is $\Pi_{2}^{P}$-complete.

Next, we prove that Minmax-3DM and its $c$-approximation version are $\Pi_{2}^{P}$ complete. In order to do this, we need the $\Pi_{2}^{P}$-completeness result on a stronger version of the problem MinmaX-Sat.

Minmax-Sat-YB: given $F(X, Y)$, with the number of occurrences of each $y \in Y$ bounded by a constant $b$, find $f_{\mathrm{SAT}-\mathrm{YB}}(F)=f_{\mathrm{SAT}}(F)$.

The more general case of Minmax-SAT-B, in which the number of occurrences of all variables in $X$ or $Y$ are bounded, is also $\Pi_{2}^{P}$-complete. Its proof is more involved and is given in a separate paper [7]. Here we give a sketch for the $\Pi_{2}^{P}$-completeness of this simpler case MinmaX-Sat-YB.

Theorem 13 Minmax-Sat-YB is $\Pi_{2}^{P}$-complete.

Proof. (Sketch) The proof is a simple modification of the reduction from the maximum satisfiability problem to the bounded-occurrence maximum satisfiability problem in [10]. It was shown in [10] that there exist a polynomial-time computable function $f$ and two integers $\alpha, b>0$ such that

(i) for each 3-CNF formula $F(X)$ with $m$ clauses, $f(F(X))=F^{\prime}\left(X^{\prime}\right)$ is a 3 -CNF boolean formula with $(\alpha+1) m$ clauses in which each variable occurs at most $b$ times, and

(ii) $\max _{\tau^{\prime}: X^{\prime} \rightarrow\{0,1\}} t c\left(F^{\prime}\left(\tau^{\prime}\right)\right)=\alpha m+\max _{\tau: X \rightarrow\{0,1\}} t c(F(\tau))$,

Now, for each $F(X, Y)$, we treat all $x \in X$ as constants and compute $f(F(X, Y))=F^{\prime}\left(X, Y^{\prime}\right)$. Then, we have

$$
\begin{aligned}
\min _{\tau_{1}: X \rightarrow\{0,1\}} \max _{\tau_{2}^{\prime}: Y^{\prime} \rightarrow\{0,1\}} t c\left(F^{\prime}\left(\tau_{1}, \tau_{2}^{\prime}\right)\right)=\min _{\tau_{1}: X \rightarrow\{0,1\}}\left[\alpha m+\max _{\tau_{2}: Y \rightarrow\{0,1\}} t c\left(F\left(\tau_{1}, \tau_{2}\right)\right)\right] \\
=\alpha m+\min _{\tau_{1}: X \rightarrow\{0,1\}} \max _{\tau_{2}: Y \rightarrow\{0,1\}} t c\left(F\left(\tau_{1}, \tau_{2}\right)\right) .
\end{aligned}
$$

Thus, if $\left\langle f_{\mathrm{SAT}}:|F|,(1-\epsilon)|F|\right\rangle$ is $\Pi_{2}^{P}$-hard, then $\left\langle f_{\mathrm{SAT}-\mathrm{YB}}:|F|,(1-\epsilon /(\alpha+1))|F|\right\rangle$ is also $\Pi_{2}^{P}$-hard.

Theorem 14 Minmax-3DM is $\Pi_{2}^{P}$-complete.

Proof. We will construct a G-reduction from Minmax-Sat-YB to Minmax-3DM. It is a modification of the L-reduction from the maximum satisfiability problem with 
bounded occurrences of variables to the maximum 3DM problem [4]. We first give a brief review of that proof.

Let $F(X)=C_{1} \wedge \ldots \wedge C_{n}$ be a 3-CNF boolean formula over variables $Y=$ $\left\{y_{1}, \ldots, y_{s}\right\}$. Let $d_{i}$ be the number of occurrences of $y_{i}$ or $\bar{y}_{i}$ in $F$. (Assuming that each clause $C_{\ell}$ has exactly 3 literals, we have $\sum_{i=1}^{s} d_{i}=3 n$.) We assume that $d_{i} \leq b$ for all $i=1, \ldots, s$. Let $M$ be the minimum number greater than $3 b / 2+1$ such that $M$ is a power of 2 . We describe below a collection $S$ of 3-element subsets of a set $W$ (called triples), without explicitly writing down all the names of elements in $W$.

(1) For each variable $y_{i}$, define $M$ identical sets of ring triples. For each $y_{i}$ and each $k, 1 \leq k \leq M$, the ring $R_{i, k}$ contains two sets of triples:

$$
\begin{aligned}
& R_{i, k}^{1}=\left\{\left\{\bar{y}_{i}[j, k], a_{i}[j, k], b_{i}[j, k]\right\}: 1 \leq j \leq d_{i}\right\}, \\
& R_{i, k}^{0}=\left\{\left\{y_{i}[j, k], b_{i}[j, k], a_{i}[j+1, k]\right\}: 1 \leq j \leq d_{i}\right\},
\end{aligned}
$$

where $j+1$ in $a_{i}[j+1, k]$ is the addition modulo $d_{i}$. This ring is the basic component of the reduction from SAT to 3DM in, e.g., [3]. We show it in Figure 6(a). Here $a_{i}[j, k]$ and $b_{i}[j, k]$ are local elements and appear in no other triples. Thus, any complete matching must take all triples in $R_{i, k}^{1}$ or all triples in $R_{i, k}^{0}$, corresponding to setting all occurrences of $y_{i}$ true or all false.

(2) For each variable $y_{i}$ and each $j, 1 \leq j \leq d_{i}$, construct two sets of tree triples: $T_{i, j}^{0}$ and $T_{i, j}^{1}$. Set $T_{i, j}^{1}$ forms a tree of size $2 M-1$ with leaves $y_{i}[j, k], 1 \leq k \leq M$, and set $T_{i, j}^{0}$ with leaves $\bar{y}_{i}[j, k], 1 \leq k \leq M$. We show a tree $T_{i, j}^{1}$ in Figure 6(b). All the internal nodes of the trees, except the roots, are the local elements. The root of $T_{i, j}^{1}$ is called $u_{i}[j]$ and the root of $T_{i, j}^{0}$ is called $\bar{u}_{i}[j]$. It is shown in [4] that, a maximum matching must take, for any $i$ and $j$, all $y_{i}[j, k]$ 's by ring triples or all by tree triples. For instance, in Figure 6, with $d_{i}=4$ and $M=8$, a maximum matching must take all circled triples or all noncircled triples. In other words, for each $i, 1 \leq i \leq s$, the maximum matching will match all ring triples and tree triples so that only all $u_{i}[j]$ 's are left free or only all $\bar{u}_{i}[j]$ 's are left free. If the matching leaves $u_{i}[j]$ 's free, then we say it corresponds to the truth assignment that sets $y_{i}$ true. For instance, the matching taking all circled triples in Figure 6 corresponds to assigning $y_{i}$ false.

(3) Identify $u_{i}[j]$ with the $j$ th occurrence of $y_{i}$, and identify $\bar{u}_{i}[j]$ with the $j$ th occurrence of $\bar{y}_{i}$. For each clause $C_{\ell}$, define 3 clause triples: $\left\{s_{1}[\ell], s_{2}[\ell], w\right\}$, where $w$ ranges over the three roots of the trees $T_{i, j}^{0}$ and $T_{i, j}^{1}$ that are identified as above to the three literals in $C_{\ell}$.

(4) Define garbage triples $\left\{g_{2 q-1}, g_{2 q}, u_{i}[j]\right\}$ and $\left\{g_{2 q-1}, g_{2 q}, \bar{u}_{i}[j]\right\}$ for all $q=$ $1, \ldots, 2 n$ and all $i=1, \ldots, s$ and all $j=1, \ldots, d_{i}$.

The above are all triples. Some simple calculation shows that there are totally $18 n M$ elements in $W$ and the number of matching is at most $6 n M$ that covers all elements. We let $K=6 n M$.

We now show that the reduction is correct. From the remarks in (1) and (2) above, we see that the maximum matchings on ring triples and tree triples correspond one-to-one with the truth assignments on $Y$. So, if $F$ is satisfied by a truth assignment $\tau$ on $Y$, then we select disjoint triples from ring triples and tree triples so that for each $y_{i}$ with $\tau\left(y_{i}\right)=1$, only the nodes $u_{i}[j]$ 's are left free, and for each $y_{i}$ with $\tau\left(y_{i}\right)=0$, only the nodes $\bar{u}_{i}[j]$ 's are left free. It can be checked that there are $(6 M-3) n$ such triples. For each clause $C_{\ell}$, we select the clause triple that covers 


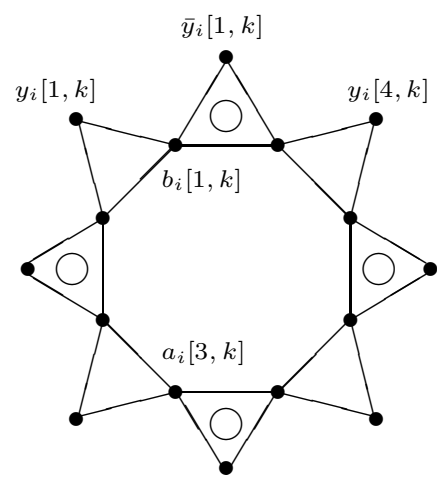

(a)

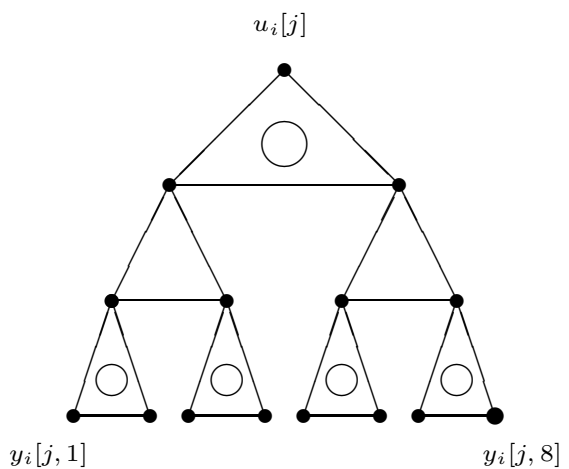

(b)

Fig. 6. (a) Ring triples with $d_{i}=4$. (b) Tree triples with $M=8$. Each triangle denotes a triple.

the root that corresponds to a true literal in $C_{\ell}$. Finally, we cover all other roots by garbage clauses. This is a complete matching of size $6 n M$.

Conversely, if there is a matching that covers every element, then it must contain for each clause $C_{\ell}$ a clause triple $\left\{s_{1}[\ell], s_{2}[\ell], w\right\}$, where $w$ is a free root note and also corresponds to a literal in $C_{\ell}$. By the property of the maximum matchings discussed in (2) above, we can define truth assignment $\tau$ on $Y$ to make all such literals true and so to satisfy $F$.

Now we describe our modification for Minmax-3DM. First, we divide $W$ into $n$ groups $W_{1}, \ldots, W_{n}$, with each $W_{\ell}$ containing all elements of $W$ that occur in the ring triples and tree triples related to clause $C_{\ell}$. More specifically, suppose the $j$ th occurrence of $y_{i}$ or $\bar{y}_{i}$ is in $C_{\ell}$, then $W_{\ell}$ contains all elements in the trees $T_{i, j}^{0}$ and $T_{i, j}^{1}$, plus the internal elements $a_{i}[j, k]$ and $b_{i}[j, k]$ for all $k \leq K$. In addition, $W_{\ell}$ contains $s_{1}[\ell], s_{2}[\ell]$ and $g_{4 \ell-p}, p=0,1,2,3$. For each $\ell, 1 \leq \ell \leq n$, we have some local triples that contain only elements in $W_{\ell}$ and inter-group triples that contain some elements in $W_{\ell}$ and some not in $W_{\ell}$. For instance, all tree triples and clause triples are local, some ring triples are local and some ring triples and garbage triples are inter-group.

Now, suppose $F(X, Y)=C_{1} \wedge \ldots \wedge C_{n}$ is a 3-CNF formula over two variable sets $X=\left\{x_{1}, \ldots, x_{r}\right\}$ and $Y=\left\{y_{1}, \ldots, y_{s}\right\}$, with each variable $y_{i}$ of $Y$ occurring in $F$ at most $b$ times. As explained in the proof of Theorem 10, we may assume that each clause $C_{\ell}$ contains at most one $X$-literal. We treat the variables in $X$ as constants, and define the triples as above from $F$, and divide them into groups $W_{\ell}$, $\ell=1, \ldots, n$. (Note that for each clause $C_{\ell}$ with an $X$-literal, it has only 2 clause triples of the form $\left\{s_{1}[\ell], s_{2}[\ell], w\right\}$.) Next, for each $1 \leq \ell \leq n$ and each $m=0,1$, we define $W_{\ell, m}$ to be a copy of $W_{\ell}$; i.e., for each element in $W_{\ell}$, attach an additional index $m$ to it (so, e.g., $s_{1}[\ell]$ becomes $s_{1}[\ell, 0]$ in $W_{\ell, 0}$ ). Then, for each group $W_{\ell, m}$, we add elements $\alpha_{\ell, m}[k], \beta_{\ell, m}[k], \gamma_{\ell, m}[k]$, for $k=1, \ldots, n$. If $C_{\ell}$ has an $X$-literal which is positive, we add one more element $\sigma_{\ell}$ to $W_{\ell, 1}$, else we add it to $W_{\ell, 0}$. We define the set $S^{\prime}$ as follows: 
(1) For each local triple in $W_{\ell}$, we include its copies in both $W_{\ell, 0}$ and $W_{\ell, 1}$ in $S^{\prime}$.

(2) For each inter-group triple between $W_{\ell}$ and $W_{\ell^{\prime}}$, we include all its copies between $W_{\ell, m}$ and $W_{\ell^{\prime}, m^{\prime}}$, for all $m, m^{\prime}=0,1$, in $S^{\prime}$.

(3) For each $C_{\ell}$, if $x_{i}$ is a literal of $C_{\ell}$, then add a triple $\left\{s_{1}[\ell, 1], s_{2}[\ell, 1], \sigma_{\ell}\right\}$ to $S^{\prime}$; if $\bar{x}_{i}$ is a literal of $C_{\ell}$, then add a triple $\left\{s_{1}[\ell, 0], s_{2}[\ell, 0], \sigma_{\ell}\right\}$ to $S^{\prime}$;

(4) We say two pairs $(\ell, m)$ and $\left(\ell^{\prime}, m^{\prime}\right)$ are inconsistent if both $C_{\ell}$ and $C_{\ell^{\prime}}$ have the same $X$-literal but $m \neq m^{\prime}$ or if $C_{\ell}$ and $C_{\ell^{\prime}}$ have the complementary $X$ literal but $m=m^{\prime}$. If $(\ell, m)$ and $\left(\ell^{\prime}, m^{\prime}\right)$ are inconsistent, then we add the triples $\left\{\alpha_{\ell, m}[k], \beta_{\ell, m}[k], \gamma_{\ell^{\prime}, m^{\prime}}[k]\right\}$ to $S^{\prime}$ for all $k=1, \ldots, n$.

Finally, we let $K=6 n M$, and claim that the reduction is correct.

First, assume that $F(X, Y) \in \mathrm{SAT}_{2}$, and let $t$ be a function from $\{1, \ldots, n\}$ to $\{0,1\}$. We check that there are at least $6 n M$ matchings in $W_{t}=\bigcup_{\ell=1}^{n} W_{\ell, t(\ell)}$. First, as in the original reduction (from SAT to $3 \mathrm{DM}$ ), we can select $(6 M-1) n$ disjoint triples from ring triples, tree triples and garbage triples. Suppose for some $\ell, \ell^{\prime}$, $(\ell, t(\ell))$ and $\left(\ell^{\prime}, t\left(\ell^{\prime}\right)\right)$ are inconsistent. Then, we can get from (4) above at least $n$ disjoint triples to make a matching of at least $6 n M$ triples. Suppose $t$ is consistent. Then, it defines a truth assignment $\tau_{1}$ on $X$ and for this $\tau_{1}$ there is a truth assignment $\tau_{2}$ on $Y$ satisfying $F$. It follows from the analysis of the original reduction that there is a matching of $6 n M$ triples. Note that for each clause $C_{\ell}$ if $\tau_{1}$ satisfies $C_{\ell}$, then the corresponding $W_{\ell, t(\ell)}$ must contain $\sigma_{\ell}$ and $\left\{s_{1}[\ell, t(\ell)], s_{2}[\ell, t(\ell)], \sigma_{\ell}\right\}$ must be in $S^{\prime}$.

Conversely, if $F(X, Y) \notin \mathrm{SAT}_{2}$ then there exists a truth assignment $\tau_{1}$ on $X$ such that $F\left(\tau_{1}, Y\right)$ is not satisfiable. Choose the corresponding $t$, i.e., $t(\ell)=1$ if and only if $\tau$ sets the $X$-literal in $C_{\ell}$ true. This function $t$ must be consistent and so there is no triple from the extra elements such as $\alpha_{\ell, m}[k]$. The only triples are the copies of those in the original reduction, and there are less than $6 n M$ disjoint triples.

Corollary 15 There exists a constant $c>1$ such that the c-approximation problem for Minmax-3DM is $\Pi_{2}^{P}$-complete.

Proof. We observe that the original reduction (from SAT to 3DM), preserves the optimum solution in the following sense: if the maximum number of satisfiable clauses is $\beta$, then the maximum matching has $(6 M-1) n+\beta n$ triples [4]. The main idea was that the design of the tree triples forces the maximum matching to make consistent truth assignments to the different occurrences of $y_{i}$. In the new reduction, this property is preserved if the function $t$ is consistent. (If $t$ is not consistent, then there are always at least $6 n M$ disjoint triples.)

For each instance $(W, S)$ of Minmax-3DM with $W$ partitioned into subsets $W_{\ell, m}$, with $1 \leq \ell \leq I, 1 \leq m \leq J$, let $\operatorname{size}(W, S)=6 M I$. Then, the above observation shows that the new reduction is a G-reduction from $\left\langle f_{\mathrm{SAT}-\mathrm{YB}}:|F|,(1-\epsilon)|F|\right\rangle$ to $\left\langle f_{3 \mathrm{DM}}: \operatorname{size}(W, S),(1-\epsilon / 6 M) \operatorname{size}(W, S)\right\rangle$.

\section{Conclusion and Open Questions}

We have demonstrated a number of min-max optimization problems to be $\Pi_{2}^{P}$ complete. Using the idea of parameterized inputs, there are apparently many more 
similar results on the generalization of $N P$-complete problems. For instance, the $\Pi_{2}^{P}$ completeness results also hold for the generalized knapsack problem and the generalized maximum set covering problem. It is hoped that these new $\Pi_{2}^{P}$-completeness results are useful for proving other natural problems such as GRN to be complete for $\Pi_{2}^{P}$ or $\Sigma_{2}^{P}$.

Although the $\Pi_{2}^{P}$-completeness results for the min-max optimization problems appear easy to prove, the corresponding $\Pi_{2}^{P}$-completeness results for the $c$ approximation problems are harder to get. We were successful only for a few such problems. It would be interesting to develop techniques for classifying the complexity of the $c$-approximation problem of the min-max problems (like the class MAX SNP for problems of the form MAX-A). In particular, it would be interesting to know whether the $c$-approximation problems of $f_{\mathrm{CIRCUIT}}$ and $f_{\mathrm{VC}}$ are $\Pi_{2}^{P}$-complete.

\section{References}

1. A. Arora, C. Lund, R. Motwani, M. Sudan and M. Szegedy, Proof verification and hardness of approximation problems, Proceedings, 33rd IEEE Symposium on Foundations of Computer Science (1992), 14-23.

2. A. Condon, J. Feigenbaum, C. Lund and P. Shor, Probabilistically checkable debate systems and approximation algorithms for PSPACE-hard functions, Proceedings, 25th ACM Symposium on Theory of Computing (1993), 305-314.

3. M. R. Garey and D. S. Johnson, Computers and Intractability: A Guide to the Theory of NP-Completeness, Freeman, San Francisco, 1979.

4. V. Kann, Maximum bounded 3-dimensional matching is MAX SNP-complete, Inform. Proc. Lett. 37 (1991), 27-35.

5. M. Kiwi, C. Lund, A. Russell, D. Spielman and R. Sundaram, Alteration in Interaction, Proceedings, 9th Structure in Complexity Theory Conference, IEEE (1994), 294-303.

6. K. Ko and C.-L. Lin, Non-approximability in the polynomial-time hierarchy, Tech. Report TR-94-2, Department of Computer Science, State University of New York at Stony Brook, Stony Brook, 1994.

7. K. Ko and C.-L. Lin, On the longest circuit in an alterable digraph, preprint, 1994.

8. K. Ko and W.-G. Tzeng, Three $\Sigma_{2}^{P}$-complete problems in computational learning theory, comput. complex. 1 (1991), 269-301.

9. C. H. Papadimitriou and K. Steiglitz, Combinatorial Optimization: Algorithms and Complexity, Prentice-Hall, Englewood Cliffs, New Jersey, 1982.

10. C. H. Papadimitriou and M. Yannakakis, Optimization, approximation, and complexity classes, J. Comput. System Sci. 43 (1991), 425-440.

11. C. H. Papadimitriou and M. Yannakakis, The traveling salesman problem with distances one and two, Math. Oper. Res. 18 (1993), 1-11.

12. Y. Sagiv and M. Yannakakis, Equivalences among relational expressions with the union and difference operations, J. Assoc. Comput. Mach. 27 (1980), 633-655.

13. L. J. Stockmeyer, The polynomial-time hierarchy, Theoret. Comput. Sci. 3 (1977), 1-22.

14. K. W. Wagner, The complexity of combinatorial problems with succinct input representation, Acta Inform. 23 (1986), 325-356. 